\title{
La rétroaction pédagogique en médecine : description et évaluation préliminaire d'un dispositif de formation sur internet pour les médecins cliniciens
}

\author{
Educational feedback in medicine: description and preliminary evaluation \\ of a web-based faculty development program for clinical educators
}

\section{Luc CôTÉ et Norma BÉLANGER}

Département de médecine familiale et de médecine d'urgence de l'université Laval et enseignants cliniciens à l'unité de médecine familiale de l'hôpital Saint-François d'Assise, Centre hospitalier universitaire de Québec, Québec, Canada

Manuscrit reçu le 23 janvier 2008; commentaires éditoriaux formulés aux auteurs le 10 novembre 2008 ; accepté pour publication le $1^{\text {er }}$ décembre 2008.

\begin{abstract}
Mots clés :
Rétroaction pédagogique ; formation professorale ; formation à distance : superviseurs en médecine

Résumé - Contexte : En médecine, la rétroaction pédagogique joue un rôle essentiel dans l'apprentissage en formation clinique à l'externat et en résidence. Ce thème est l'objet de nombreux ateliers de formation pédagogique dispensés en présence à des médecins enseignants. But : Cet article vise à décrire un dispositif de formation innovant sur la rétroaction pédagogique en médecine offert sur internet. Nous en faisons aussi une évaluation préliminaire à partir du point de vue des participants, des responsables et d'une consultante externe. Sujets/Matériel : Entre 2005 et 2006, 21 médecins superviseurs associés à l'université Laval (Québec, Canada) ont complété un dispositif de formation composé de six modules sur le Web avec un accompagnement individualisé sous forme de tutorat à distance. Résultats : $\mathrm{Ce}$ dispositif de formation représente une innovation pédagogique originale, utile et très appréciée dans son ensemble. La nature et la variété des exercices, ainsi que l'encadrement sous forme de tutorat figurent parmi les points forts. La principale difficulté vécue par les participants et les tuteurs fut d'avoir à écrire le contenu de leurs rétroactions plutôt que d'échanger verbalement avec leurs interlocuteurs. Conclusion : Diverses modifications seront introduites dans la nouvelle version du dispositif. Nous porterons une attention particulière aux moyens de mieux développer chez les médecins le transfert des apprentissages dans leur pratique quotidienne.
\end{abstract}

Keywords: Educational feedback; faculty development; online formation; clinical educators

Abstract - Context: In medicine, educational feedback plays an essential role for learning during clerkship and residency clinical trainings. This matter is the object of numerous educational formation workshops given to clinical educators. Objectives: To describe an innovative web-based faculty development program offered on the Internet based on educational feedback in medicine and to preliminary evaluate it from the point of view of participants, leaders and an external consultant. Subjects/Material: Between 2005 and 2006, 21 clinical educators associated to the University of Laval (Québec, Canada) completed the web-based training tool, which comprised six modules on the Internet accompanied with an 
individualized e-tutorial. Results: This web-based training tool represents an original, useful and highly appreciated educational innovation. The nature and the variety of practical exercises with the combination of the individualized e-tutorial were both reported to be key points of the training device. The main difficulty reported by participants and tutors was to write the content of feedback instead of giving it orally. Conclusion: Diverse improvements will be included in the new version of the web-based training tool. We will put our attention on the ability of clinical educators to give an educational feedback in their daily practice.

\section{Introduction}

En formation clinique, les externes et les résidents apprennent à exercer leur profession principalement au contact des patients, en observant leurs superviseurs dans divers contextes ainsi qu'en discutant avec eux lors de périodes de rétroaction (feedback). La rétroaction pédagogique est une discussion entre le superviseur et l'étudiant, laquelle est axée sur la performance de l'étudiant. Cette performance est jugée en fonction des objectifs d'apprentissage et du niveau de performance attendu dans un contexte donné. La rétroaction échangée, en contexte d'évaluation formative ou sommative, vise à guider et soutenir l'étudiant dans le développement de ses compétences professionnelles. La rétroaction joue un rôle essentiel car, selon la perspective théorique développée par Ericsson et al. sous la dénomination de «pratique délibérée et intentionnelle » (deliberate practice), le développement de l'expertise dépend non seulement de la qualité et de la quantité des efforts consacrés à apprendre mais de la qualité et de la fréquence de la rétroaction reçue en cours d'apprentissage ${ }^{[1,2]}$.

Des revues de la littérature en formation clinique en médecine ont démontré que la capacité de fournir une rétroaction pédagogique soutenue par des échanges judicieux avec l'étudiant était une des caractéristiques des enseignants cliniciens jugés excellents ${ }^{[3-5]}$. Lors de la discussion de cas avec l'étudiant, ceux-ci visent le double objectif de vérifier la qualité des soins au patient ainsi que de la qualité de l'apprentissage de l'étudiant. Les caractéristiques d'une rétroaction favorisant l'apprentissage ${ }^{[6-8]}$, ainsi que plusieurs modèles ${ }^{[9-11]}$ et stratégies favorisant l'apprentissage en contexte clinique ${ }^{[12-16]}$, ont été décrits.

Or, même si un superviseur connaît les principes d'une bonne rétroaction (par exemple, formuler successivement et de façon équilibrée les points positifs et ceux à améliorer), divers facteurs associés à l'étudiant, au superviseur et au contexte, vont influencer le contenu et la forme de la rétroaction échangée. Par exemple, certains superviseurs sont très habiles pour cibler et discuter les aspects de la performance à améliorer chez l'étudiant alors que d'autres excellent dans l'art de renforcer leurs points positifs. De même, pour diverses raisons, le degré d'ouverture de l'étudiant et son aptitude à recevoir de la rétroaction varient considérablement. Enfin, les contraintes de temps inhérentes au travail en milieu clinique, surtout en contexte de travail à haut débit, vont influencer le choix du contenu de la rétroaction. La question se pose alors de savoir comment partager avec l'étudiant une rétroaction qui valorise ses forces et comment utiliser ces dernières comme points d'appui contribuant à l'aider à corriger ses points faibles. C'est notamment le cas lors de rétroactions se rapportant à des situations cliniques où la performance nécessite d'être corrigée de manière majeure.

La seule revue de littérature publiée sur la formation pédagogique des enseignants en médecine ${ }^{[17]}$ montre que la majorité des formations s'adressent à des médecins impliqués dans la formation clinique, surtout en médecine interne et en médecine familiale et que les thèmes de la rétroaction et de l'évaluation figurent parmi les plus fréquents. Les formations consistent habituellement en des ateliers en face-à-face, d'une durée variant entre 
quelques heures et quelques jours et parfois en des programmes de plus longue durée. À notre connaissance, aucune formation structurée à partir d'une série de modules proposés sur internet et portant spécifiquement sur la rétroaction en médecine n'est proposée

Cet article a pour objectif : a) de décrire un dispositif de formation innovant dédié à la rétroaction pédagogique en médecine et offert intégralement en ligne sur internet; b) d'en rapporter une évaluation préliminaire à partir des opinions des médecins ayant bénéficié de cette formation, de la réflexion des responsables, ainsi que de l'analyse faite par une consultante externe.

\section{Matériel et méthodes}

\section{Description du dispositif pédagogique}

\section{Contexte et clientèle}

Depuis quelques années, le réseau d'enseignement clinique en médecine de l'université Laval (Québec, Canada) est de plus en plus dispersé sur un vaste territoire et doit composer avec une augmentation significative des cohortes d'externes et de résidents. C'est dans ce contexte qu'en 2004, une équipe du centre de développement pédagogique de la faculté de médecine a développé le programme de formation intitulé «La rétroaction qui favorise l'apprentissage : le cœur de la formation clinique ». Il s'agit d'une formation pédagogique mise en ligne sur internet destinée à des médecins enseignants impliqués dans l'encadrement clinique, en particulier auprès des externes. Depuis quelques années, ce centre offre à quelques reprises pendant l'année une formation sur la rétroaction en mode face-à-face. Toutefois, plusieurs superviseurs, surtout ceux exerçant en régions géographiquement éloignées, ne peuvent y participer en raison des contraintes liées à l'horaire fixe des formations, ainsi qu'au temps et aux coûts associés aux déplacements. Pour ces raisons pratiques, le projet de formation sur internet devenait pertinent. Par ailleurs, des raisons pédagogiques ont aussi influencé le choix d'une formation à distance. En effet, il existe une abondante littérature mettant en évidence les avantages de la formation à distance comparée à celle en présence, pour autant qu'elle soit bien planifiée et élaborée en conséquence. Parmi les avantages, mentionnons l'autonomie dans les apprentissages, le développement de la pratique réflexive, l'apprentissage individualisé adapté à divers styles d'apprentissage à partir de méthodes interactives et variées, ainsi que l'apprentissage collaboratif ${ }^{[18-20]}$.

\section{Objectifs}

La formation vise cinq objectifs : 1) reconnaître l'importance de la rétroaction dans la formation clinique ; 2) décrire les caractéristiques d'une rétroaction utile à l'apprentissage ; 3) élaborer une démarche efficace de rétroaction ; 4) échanger des rétroactions en divers contextes de stage et lors de la remise de l'évaluation de mi-stage; 5) développer des habiletés de pratique réflexive sur la rétroaction.

\section{Contenus et modalités d'apprentissage}

La formation consiste en six modules disponibles en ligne sur le Web (plate-forme Typo 3) que les participants complètent individuellement, à leur rythme. Ces modules, axés sur diverses dimensions de la rétroaction, sont élaborés selon une séquence d'activités représentatives du déroulement d'un stage clinique : de l'accueil du stagiaire jusqu'à la remise de l'évaluation à mi-stage; les aspects liés à l'évaluation terminale de fin de stage faisant l'object d'une autre formation en préparation. Les titres des modules sont : 1) On se présente, on se prépare ; 2) Bien accueillir l'externe : une différence pour la rétroaction; 3) La rétroaction qui favorise l'apprentissage : qu'est-ce que ça signifie ? 4) Les situations de rétroaction plus complexes; 5) L'évaluation à mi-stage : une démarche qui se planifie ; 6) L'évaluation à mistage : une démarche interactive de rétroaction.

Les contenus des modules se réfèrent principalement aux éléments suivants : les concepts de supervision, de rétroaction et d'évaluation; les facteurs 
associés au confort et à l'inconfort à échanger de la rétroaction; les caractéristiques de la rétroaction qui favorise l'apprentissage ; les stratégies pour partager de la rétroaction dans différents contextes, plus particulièrement lors de la remise d'une évaluation formative à mi-stage.

Les modalités pédagogiques ont été choisies en fonction des principes d'apprentissage issus de la théorie de l'apprentissage par l'expérience ${ }^{[21]}$, de la psychologie cognitive ${ }^{[13,22]}$, de la pratique réflexive ${ }^{[23]}$, de la théorie de l'apprentissage social ${ }^{[24]}$ ainsi qu'en fonction des recommandations sur le développement d'un programme ${ }^{[25]}$ et le développement d'activités de formation à distance $^{[26,27]}$. Par conséquent, nous avons été attentifs à : a) situer les apprentissages à partir de situations représentatives de la pratique de la supervision pendant l'externat en médecine ; b) solliciter l'implication active des participants par des exercices de complexité variable favorisant l'arrimage des nouvelles connaissances à leurs connaissances antérieures et le transfert des apprentissages ; c) inciter les participants à partager périodiquement avec nous leurs expériences, leurs incertitudes et leurs questionnements ; d) mettre les participants en situation de s'auto-évaluer régulièrement ; e) partager régulièrement de la rétroaction avec les participants et ainsi jouer le rôle de modèle potentiel auprès d'eux dans l'art d'échanger de la rétroaction.

Une autre modalité pédagogique majeure de cette formation est que chaque participant est jumelé à un tuteur (LC ou NB) dès le début. Le participant est invité à lui acheminer ses contributions correspondant aux activités prévues pour les différentes tâches du module dès qu'elles sont effectuées. Le tuteur échange régulièrement par courriel et, dans un délai de moins de 48 heures, fournit de la rétroaction sur les exercices effectués, stimule la réflexion, répond aux questions, réagit aux commentaires, etc. Cet accompagnement a deux raisons d'être. La première consiste à créer et à développer une « relation d'apprentissage » avec les participants car, de par la nature même de l'apprentissage à partir des ressources médiatisées par internet, les contacts sont «virtuels ». La seconde est qu'en recevant périodiquement diverses rétroactions de son tuteur, chaque participant a la possibilité d'analyser le contenu et la manière dont la rétroaction a été transmise et d'y réagir. Cette modalité a le potentiel d'enrichir les options d'interventions du participant en fonction des cas de supervision présentés dans les modules ou des cas réels rencontrés dans leur milieu clinique.

\section{Aperçu des modules de formation}

Sur la page d'accueil de chaque module apparaissent les objectifs et les activités du module, ainsi qu'un rappel du contenu général du ou des modules précédents (figure 1). Les modules comportent des exercices individuels, de brèves capsules d'information, des occasions de s'auto-évaluer ainsi que l'accès à des outils pédagogiques, à des sites éducatifs ou à des lectures complémentaires. Quelques exemples d'outils pédagogiques sont présentés sur la figure 2 .

Dans les faits, les participants travaillent avec cinq stagiaires virtuels : Isabelle, David, Louise, Catherine et Bernard. Par exemple, au module 3 ( $\mathrm{La}$ rétroaction constructive, qu'est-ce que ça signifie?), les participants doivent préparer leur rétroaction à David, externe senior au service d'urgence, en tenant compte de sa performance et du contenu de la fiche d'autoévaluation qu'il a complétée à la fin de sa journée de stage (figure 3).

Pour être représentatives de la progression d'un étudiant en cours de stage, certaines mises en situations sont construites en faisant évoluer le même stagiaire d'un module à l'autre. Voici deux exemples.

Dans le cas de Louise, externe junior qui évolue bien :

- au module 4, les participants doivent lui transmettre par écrit de la rétroaction suite à une période de travail à l'unité de médecine interne ;

- au module 6, on leur demande de préparer son évaluation à mi-stage, en tenant compte des étapes et des moyens proposés au module 5 . Après avoir rédigé la fiche d'évaluation, ils comparent le contenu de leur fiche avec celle remplie 


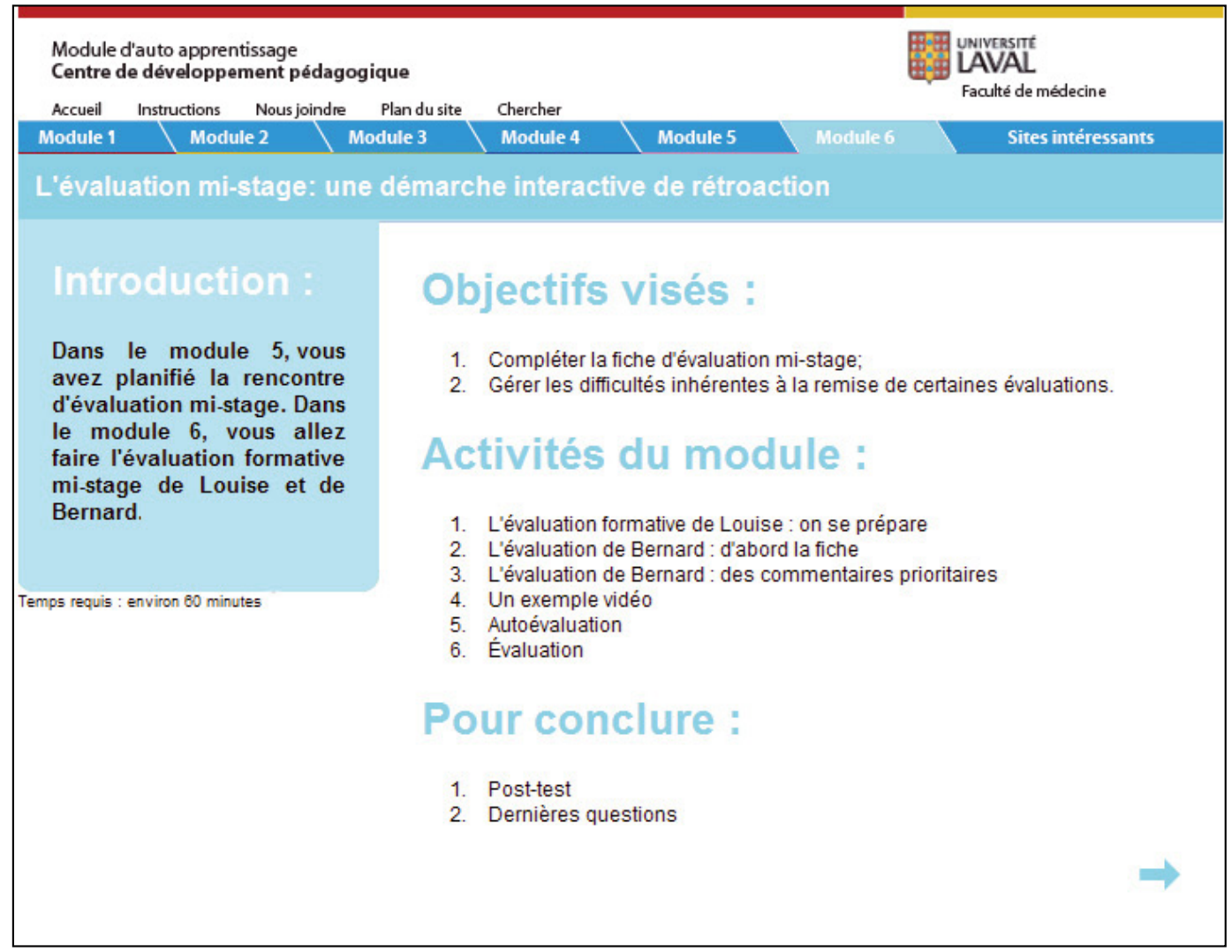

Fig. 1. Page d'accueil-type d'un module proposé en ligne sur internet dans le cadre du programme de formation à la supervision pédagogique.

par les tuteurs. Ils commentent cette dernière, puis rédigent une prescription pédagogique pour aider l'étudiante à s'améliorer en fonction d'une difficulté particulière (Figure 4);

- toujours au module 6 , ils décrivent concrètement comment ils lui remettraient cette évaluation en tenant compte des principes pédagogiques présentés au module 3 .

Avec Bernard, externe senior qui éprouve des difficultés d'apprentissage :

- au module 4, les participants doivent écrire une rétroaction dans laquelle ils nomment clairement ses difficultés à générer des diagnostics différentiels dans des situations usuelles pour son niveau de formation;

- au module 6, à partir de la synthèse de la performance de Bernard et du contenu de la fiche d'évaluation à mi-stage rédigée par les superviseurs, les participants décrivent comment ils annonceraient au stagiaire qu'il est en difficulté et quelles stratégies ils utiliseraient pour trouver avec lui des correctifs à mettre en place d'ici la fin de son stage. Les participants sont aussi invités à justifier leurs choix pédagogiques. Enfin, ils ont à commenter une vidéo démontrant une façon de remettre l'évaluation de mi-stage à Bernard.

\section{Méthodes mises en œuvre pour l'évaluation préliminaire du dispositif pédagogique}

Récemment, Barrows a rappelé que toute innovation pédagogique doit être évaluée ${ }^{[28]}$. L'évaluation préliminaire de notre dispositif de formation repose sur un processus d'évaluation qualitative continue 


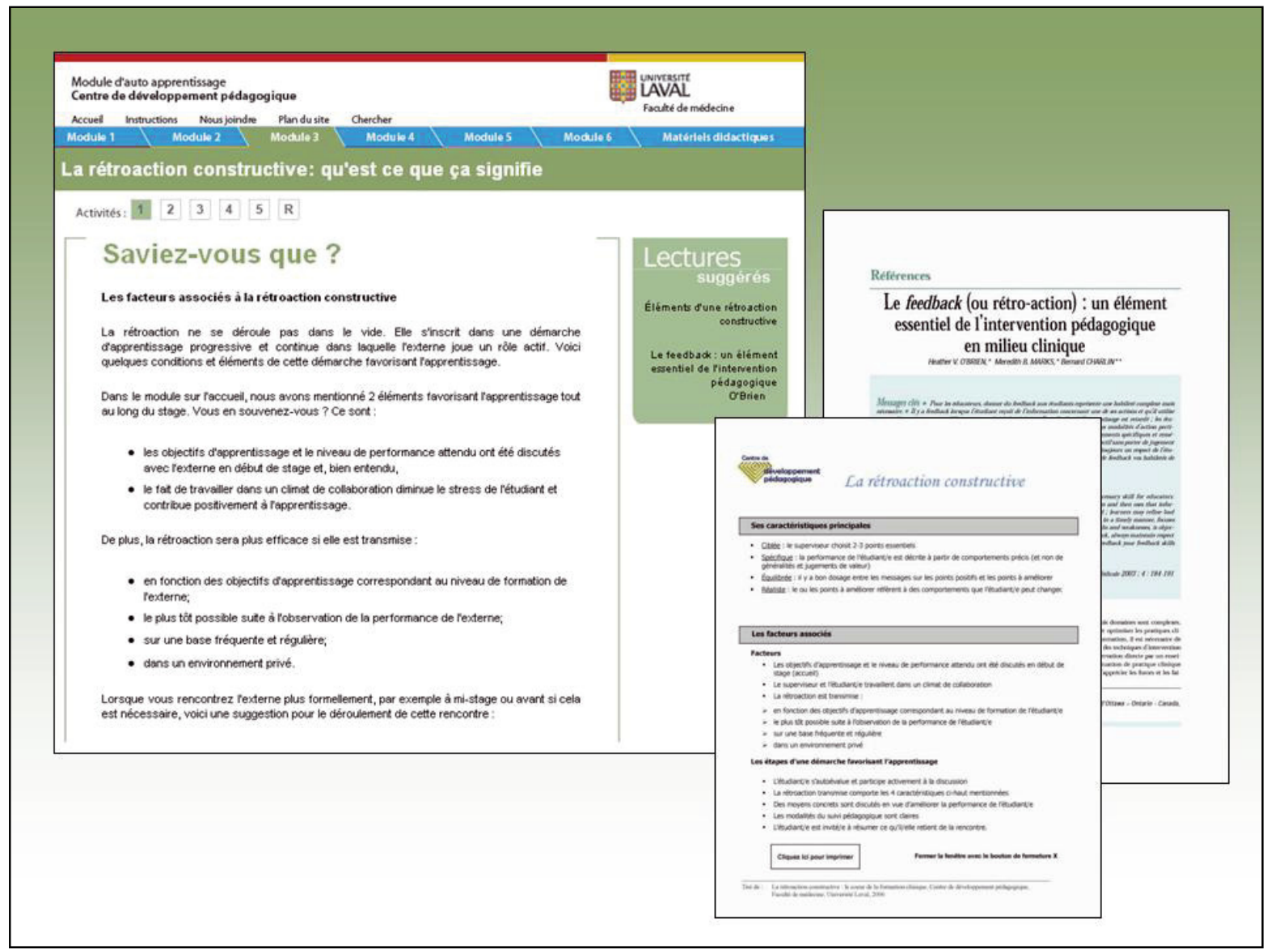

Fig. 2. Exemples d'outils pédagogiques et de ressources didactiques proposés en ligne sur internet dans le cadre du programme de formation à la supervision pédagogique.

selon les principes du modèle de pratique réflexive de Schön : réflexion dans l'action et réflexion sur l'action ${ }^{[23]}$. Trois sources d'information furent utilisées : les participants, les animateurs et une consultante externe, spécialiste des applications pédagogiques des technologies de l'information et de la communication (APTIC). Cette triangulation des sources d'information figure parmi les critères de crédibilité en recherche qualitative ${ }^{[29]}$. Le recueil des informations s'est fait selon les trois étapes suivantes :

Étape 1 : Mise à l'essai du dispositif, pendant l'hiver 2005, avec un groupe-pilote de six médecins enseignants qui ont répondu individuellement, par écrit et verbalement, à nos questions sur le contenu et la forme des modules et au sujet de la démarche d'apprentissage. Parallèlement, les animateurs ont individuellement tenu un journal de bord en consignant des observations quant à leur expérience d'animation, les contenus des modules qui semblaient bien fonctionner, les problèmes rencontrés, les questions en suspens, etc. Les résultats de cette mise à l'essai nous ont aidé à produire l'actuelle version du dispositif qui fut offert à deux reprises, soit à l'automne 2005, puis à l'automne 2006. Pour des raisons de faisabilité, et pour assurer la qualité de l'encadrement des participants par les tuteurs, le nombre maximal de participants fut limité à 12 par période de formation.

Étape 2 : À la fin de chaque module (automne 2005 et automne 2006), les participants devaient compléter individuellement un questionnaire portant en 

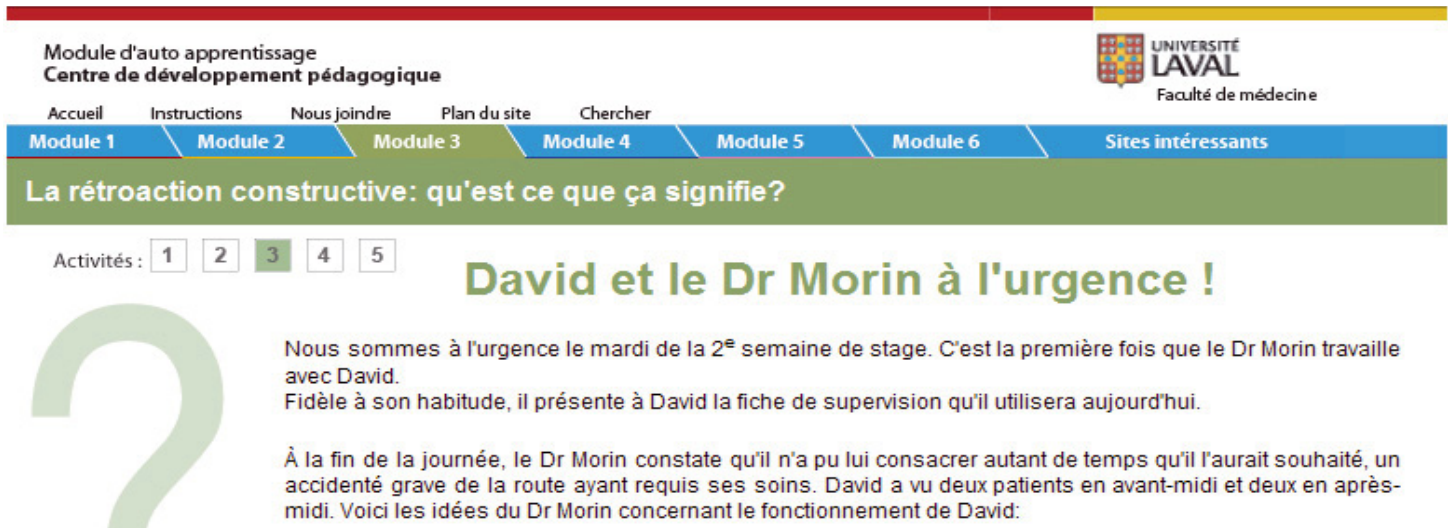
accidenté grave de la route ayant requis ses soins. David a vu deux patients en avant-midi et deux en aprèsmidi. Voici les idées du Dr Morin concernant le fonctionnement de David:

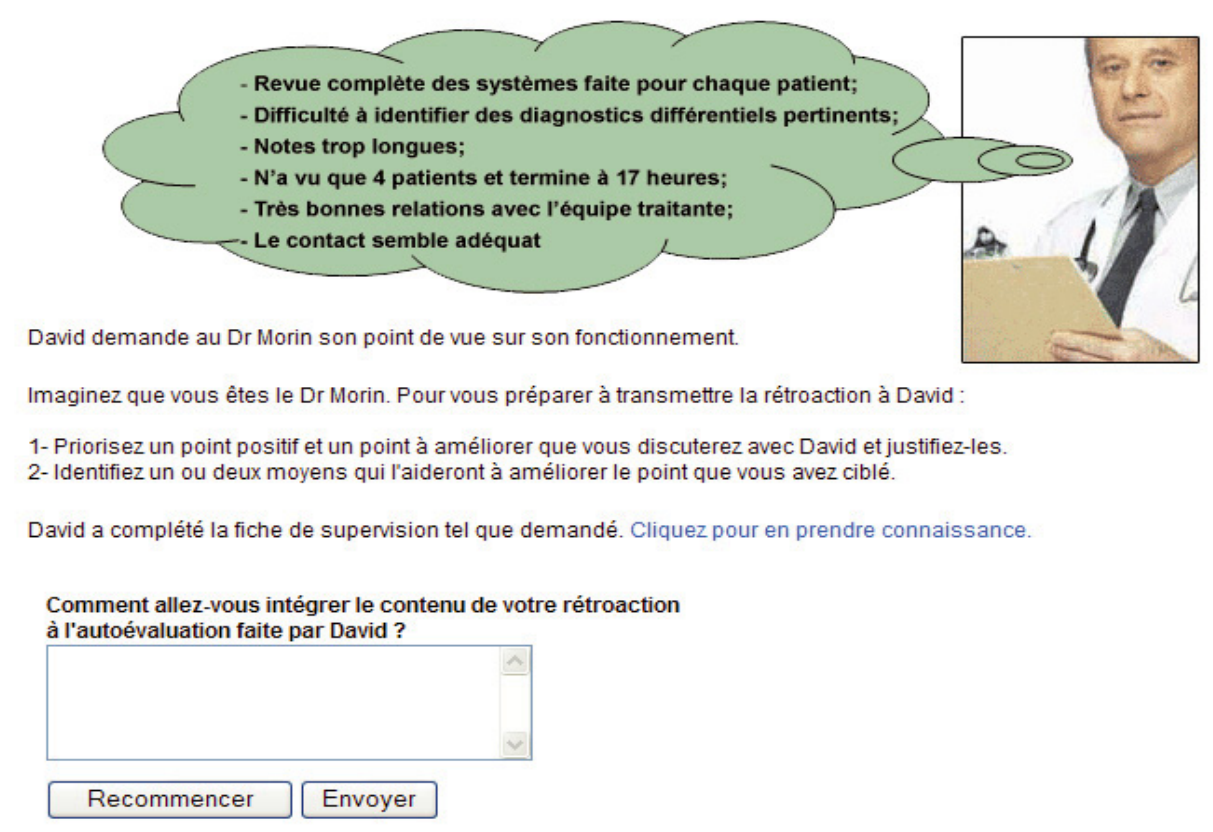

Fig. 3. Exemple d'une tâche avec David au module 3, proposée en ligne sur internet aux participants du programme de formation à la supervision pédagogique.

particulier sur les apprentissages réalisés pendant le module, les forces et limites du module ainsi que le transfert dans la pratique (comment ils projetaient d'appliquer leurs apprentissages dans leur contexte de travail). De plus, à la fin de la formation, on leur demandait d'évaluer sur le web l'ensemble de la formation, en nous indiquant plus particulièrement ce qui avait été plus aidant et difficile pour l'atteinte des objectifs visés. De plus, trois participants ont aussi communiqué verbalement avec l'un des animateurs pour discuter plus en profondeur de certains aspects de la formation. Comme à l'étape précédente, les animateurs ont tenu un journal de bord.

Étape 3 : En 2007, les animateurs ont demandé à une consultante externe, spécialiste des applications pédagogiques des technologies de l'information et de la communication (APTIC) d'analyser le dispositif. Celle-ci a rencontré les animateurs pour discuter les points forts et les limites et proposer des améliorations pour les années à venir. 


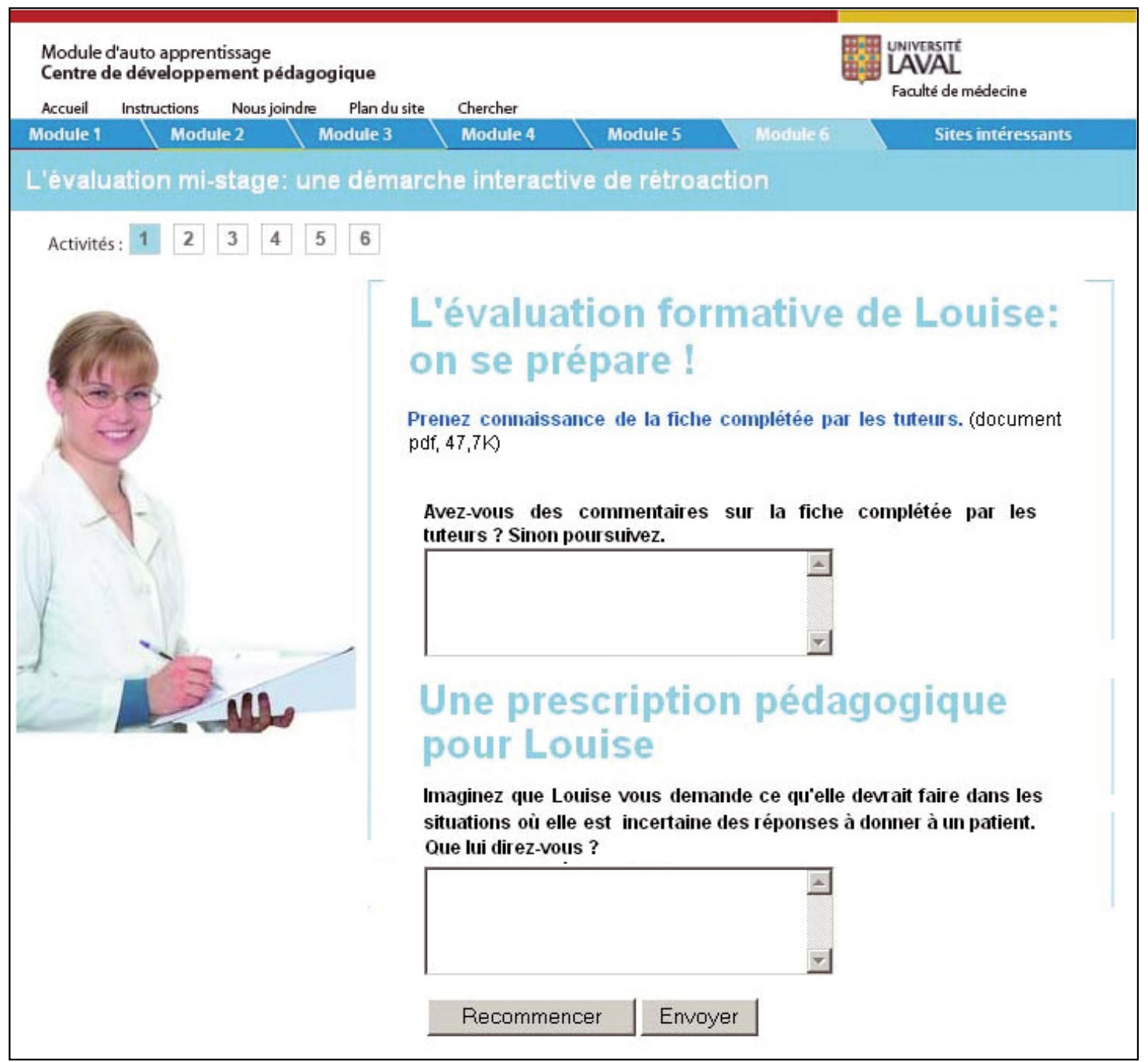

Fig. 4. Exemple d'une tâche avec Louise au module 6, proposée en ligne sur internet aux participants du programme de formation à la supervision pédagogique.

\section{Résultats}

Au total, 21 médecins (13 femmes et 8 hommes) provenant du milieu de la médecine familiale et de diverses spécialités, incluant ceux du groupe-pilote, ont achevé la formation. La moitié d'entre eux avaient moins de six années d'expérience en enseignement clinique alors que trois participants avaient plus de vingt ans d'expérience. Au départ, 29 médecins s'étaient inscrits mais huit d'entre eux (cinq femmes et trois hommes) se sont désistés en raison d'un manque de temps relié à des charges cliniques imprévues $(n=4)$ ou à des problèmes de santé $(n=2)$. Deux participants ont abandonné sans donner de raison.

Points forts du programme

\section{Point de vue des participants}

Les forces de cette formation, telles que rapportées par la majorité des participants, sont la qualité de la 
formation dans son ensemble, en particulier : a) la pertinence et la variété des mises en situation; b) l'utilité et la brièveté des capsules d'information; c) la qualité de la rétroaction avec les tuteurs; d) la navigabilité agréable et facile sur le web; e) la charge de travail raisonnable (le temps pour compléter chaque module a varié en moyenne entre 30 et 60 minutes) ; f) l'obtention de crédits de formation médicale continue.

\section{Point de vue des tuteurs}

Notre expérience de concepteurs et de tuteurs nous amène à identifier les forces suivantes. Selon nous, les deux principales forces de la formation sont d'abord le choix des exercices, dont les situations de supervision, en fonction d'une séquence d'activités représentatives du déroulement d'un stage clinique. Les situations de supervision, représentatives de la réalité clinique et évolutives quant à leur complexité, ont contribué à l'application et au transfert des apprentissages, d'un module à l'autre. En analysant le contenu des réponses des participants aux situations de supervision qui leur étaient soumises, nous avons été à même de constater que la très grande majorité semblait mieux outillée en fin qu'en début de formation, pour échanger de la rétroaction et ce, même si les situations présentées dans les derniers modules étaient plus complexes. Lors de l'évaluation du dispositif, les participants nous ont d'ailleurs exprimé le sentiment d'avoir non seulement mieux compris ce qu'était une rétroaction pédagogique mais d'avoir développé des habiletés à la partager avec les étudiants.

L'autre force est l'accompagnement longitudinal et individualisé, sous forme de tutorat, par des superviseurs d'expérience. Cette modalité d'apprentissage a permis aux tuteurs non seulement d'échanger de la rétroaction en fonction des principes dont ils faisaient la promotion dans la formation et, de ce fait, jouer le rôle potentiel de modèle, mais aussi de stimuler la réflexion pédagogique des participants en réagissant à leurs commentaires, en posant des questions, en présentant des anecdotes de situations de supervision vécues, etc. Cet encadrement individualisé a fait en sorte de créer une «alliance pédagogique » ou un lien d'apprentissage particulièrement important dans une formation sur le web.

\section{Point de vue de la consultante APTIC}

Pour la consultante, les principaux points forts de ce programme sont : a) la démarche pédagogique dans son ensemble; b) la pertinence et la variété de mises en situation authentiques; c) l'utilité et la brièveté des capsules d'information ; d) la navigabilité agréable et facile sur le web; e) le système de tutorat.

Limites du programme

\section{Point de vue des participants}

Au niveau des limites, plusieurs participants ont rapporté : a) les contraintes liées au temps pris pour écrire les réponses aux questions se rapportant aux mises en situation : ils trouvaient ardu d'écrire plutôt que de dire; b) le fait de ne pas avoir la réaction immédiate de l'étudiant avec qui ils échangeaient la rétroaction : c'est le tuteur qui réagissait aux réponses du participant ; c) l'absence de contact avec les autres participants ; d) la difficulté d'intégrer cette formation à leur horaire chargé, même si la charge de travail pour chacun des modules était jugée raisonnable.

\section{Point de vue des tuteurs}

Au niveau des limites, nous avons identifié sensiblement les mêmes que celles des participants. D'abord, la relation tuteur-participant s'est faite principalement par courriel. Bien que pour la rétroaction, le choix des mots «stimule » souvent la réflexion pédagogique, avoir à écrire le contenu d'une rétroaction peut être une entrave au nombre et à la qualité des échanges de même qu'à la qualité du lien entre le tuteur et le participant. En effet, le fait de 
devoir répondre par écrit aux questions des mises en situation exige plus de temps pour le participant; il est souvent plus facile et efficace d'expliquer verbalement plutôt que par écrit.

En ce qui concerne l'absence de contacts entre les participants, il est vrai que l'apprentissage par les pairs serait une modalité à considérer. Toutefois, lors de la mise à l'essai de notre formation, nous avions voulu intégrer un forum de discussion, ce que les participants d'alors nous avaient déconseillé. Ils jugeaient qu'ils n'auraient pas le temps de contribuer aux échanges, même en mode asynchrone.

Enfin, bien que pertinent, l'encadrement par tutorat représente une charge de travail importante pour les tuteurs, notamment si le participant a du mal à intégrer les apprentissages visés; dans ce cas, les réponses du tuteur exigent souvent davantage de réflexion et d'écriture par courriel.

\section{Point de vue de la consultante APTIC}

Les principales suggestions de la consultante visant à améliorer ce dispositif sont : a) d'intégrer, au début du dispositif, de courts exemples vidéo présentant quelques façons courantes et inappropriées de partager de la rétroaction en contexte clinique en médecine. Ceci permettrait aux participants d'analyser, d'une façon non menaçante, les principales erreurs souvent commises en éducation médicale, en fonction des caractéristiques reconnues d'une rétroaction efficace. Ces exemples serviraient d'arguments supplémentaires à l'importance de la formation pédagogique sur la rétroaction; b) d'ajouter, dans chaque module, de courtes séquences vidéos de démonstration se rapportant aux situations de supervision soumises aux participants et dans lesquels apparaîtraient les bases théoriques en appui à ce qui est démontré ; c) d'ajouter un outil permettant aux participants et aux tuteurs de transmettre les commentaires en mode audio plutôt que par écrit; d) d'ajouter une activité d'intégration à la fin du dispositif dans laquelle le participant aurait à échanger, en mode audio, une rétroaction à un externe dont le rôle serait joué par un étudiant simulé et standardisé.

\section{Discussion et conclusion}

Pour les raisons invoquées précédemment, ce dispositif de formation représente une innovation pédagogique originale, utile et très appréciée dans son ensemble. À la lumière des constats faits par les responsables et des commentaires reçus de la part des participants et de la consultante, nous devrons trouver des moyens d'améliorer son efficacité en solutionnant les problèmes identifiés. Nous travaillons déjà à donner suite aux suggestions faites, plus spécifiquement à celles de notre consultante.

Concernant le caractère préliminaire de l'évaluation du programme, nous sommes conscients que nous devrons aller au-delà des mesures de satisfaction en évaluant comment les participants intègrent les apprentissages sur la rétroaction à leur travail d'encadrement clinique au quotidien. Nous examinons différentes possibilités dont une consisterait à demander aux participants de réaliser, à diverses étapes de la formation, des enregistrements audio de rétroactions avec les étudiants qu'ils supervisent ou avec des étudiants simulés et standardisés. Les rétroactions seraient commentées par un des tuteurs. Toutefois, les contraintes de temps avec lesquelles les superviseurs doivent composer sont un facteur qu'il faudra évidemment prendre en considération. Dans une perspective plus large que celle de l'actuel dispositif, nous nous interrogeons à la fois quant au type de formations pédagogiques et cliniques et quant aux modalités de suivi longitudinal susceptibles de mieux développer chez les médecins le transfert des apprentissages dans leur pratique quotidienne. Bien que la question du transfert ne soit pas spécifique à la formation pédagogique ni à la formation à distance, elle nécessite qu'on s'y intéresse sérieusement.

\section{Contributions}

Les deux auteurs ont conçu et réalisé la formation décrite et en sont les responsables scientifiques. Ils ont collégialement rédigé et révisé ce manuscrit. 


\section{Références}

1. Ericsson KA, Krampe RT, Tesch-Römer C. The role of deliberate practice in the acquisition of expert performance. Psycho Rev 1993;100:363-406.

2. Ericsson KA, Charness N, Paul J. Feltovich PJ, Hoffman RR (eds). The Cambridge Handbook of Expertise and Expert Performance. New York: Cambridge University Press, 2006.

3. Irby, DM. Teaching and learning in ambulatory care settings: a thematic review of the literature. Acad Med 1995;70:898-931.

4. Kilminster SM, Joly BC. Effective supervision in clinical settings: a literature review. Med Educ 2000;34:827-40.

5. Bowen JL, Irby DM. Assessing quality and costs of education in the ambulatory setting: a review of the literature. Acad Med 2002;77:621-80.

6. Ende J. Feedback in clinical medical education. JAMA 1983;250:777-81.

7. Hesketh EA, Laidlaw JM. Developing the teaching instinct, 1: Feedback. Med Teach 2002;24:245-8.

8. O'Brien HV, Marks MB, Charlin B.Le feedback (ou retro-action). Un élément essentiel de l'intervention pédagogique en milieu clinique. Pédagogie médicale 2003;4:184-91.

9. Neher JO, Gordon KC, Meyer B, Stevens N. A five-step "microskills" model of clinical teaching. Jour Am Board of Fam Pract 1992;5:419-24.

10. Wolpaw TM, Wolpaw DR, Papp KK. SNAPPS : A learnercentered model for outpatient education. Acad. Med. 2003;78:893-8.

11. Lillich DW, Mace K, Goodell K, Kinnee C. Active precepting in the residency clinic: A pilot study of a new model. Fam Med 2005;37:205-10.

12. Westberg J, Jason H. Fostering learners' reflection and selfassessment. Fam Med 1994;26:278-82.

13. Gruppen LD. Implications of cognitive research for ambulatory care education. Acad Med 1997;72:117-20.

14. Connell KJ, Bordage G, Chang RW, Howard BA, Sinacore J. Measuring promotion of thinking during precepting encounters in outpatient settings. Acad Med 1999; 74 Supplement: S10-S12.

15. Branch WT, Paranjape AP. Feedback and reflection: teaching methods for clinical settings. Acad Med 2002;77:1185-8

16. Bowen JL. Educational diagnostic reasoning. New Engl J Med 2007;355:2217-25.
17. Steinert Y, Mann K, Dolmans D, Spencer J, Gelula M, Prideaux D. A systematic review of faculty development initiative designed to improve teaching effectiveness in medical education: BEME guide no. 8. Med Teach 2006; 28:497-526.

18. Compendium de références bibliographiques et de résumés sur la recherche et l'intervention en formation à distance Université du Québec, Téluq, 2004. [On-line] Disponible sur : http://www.teluq.uquebec.ca/ webteluq/extras/fad/compendium.html

19. Audet L. Réseau d'enseignement francophone à distance du Canada (REFAD). Pour franchir la distance. Guide de formation et de soutien aux enseignants et formateurs en formation à distance. [On-line] Disponible sur : http://www.refad.ca/nouveau/guide_ formateurs_FAD/guide_formateurs_FAD.html

20. La formation ouverte et à distance (F.O.A.D.). Académie de Bordeaux. [On-line] Disponible sur : http:// formation. ac-bordeaux. fr/formation/fo_ foad_ava.html

21. Dewey J. How We Think. Boston: Heath, 1933.

22. Tardif J. Pour un enseignement stratégique. Montréal : Éditions Logiques, 1992.

23. Schön DA. The reflective practitioner: how professionals think in action. New York: Basic Books, 1983.

24. Bandura A. Social learning theory. New York: General Learning Press, 1977.

25. Harden RD. Curriculum planning and development. Dans : Dent, JA et Harden, RM (eds). A Practical Guide for Medical Teachers, Edinburgh: Elsevier, 2005.

26. Réseau de valorisation de l'enseignement, Guide de conception pédagogique et graphique d'un site éducatif sur le réseau Internet. Université Laval : Québec, 2003.

27. Lebrun M. E Learning pour enseigner et apprendre : Allier pédagogie et technologie. Louvain-la-Neuve, Belgique: Academia Bruylant, 2005.

28. Barrows HS. Innovations without appropriate assessment are limited usefulness. Teach Learn Med 2008;20:287.

29. Côté L, Turgeon J. Comment lire de façon critique les articles de recherche qualitative en médecine. Pédagogie Médicale. 2002;3:81-90.

Correspondance et offprints : Luc Côté, Consortium pédagogique. Faculté de médecine, Bureau 3370, Pavillon Ferdinand-Vandry Université Laval. Québec (Québec) Canada G1K7P4

Mailto : luc.cote@fmed.ulaval.ca 